\title{
Clustering of colorectal neoplasia: characteristics of coexisting adenomas in patients with severely dysplastic polyps or invasive (malignant) polyps as compared to patients with benign adenomas or carcinomas
}

\author{
Amos Pines ${ }^{1,3}$, Leon Bat ${ }^{1}$, Eliahou Shemesh ${ }^{1}$, Joseph Rosenblum ${ }^{1}$, \\ Ada Horowitz ${ }^{2}$, Yoram Levo ${ }^{3}$ and Jose J. Bubis ${ }^{2}$
}

${ }^{1}$ Gastroenterology and ${ }^{2}$ Pathology Institutes, the Sheba Medical Center, Tel-Hashomer, and ${ }^{3}$ Department of Medicine 'T', Ichilov Hospital, Tel-Aviv Medical Center; affiliated to the Sackler Faculty of Medicine, Tel-Aviv University, Tel-Aviv, Israel

\begin{abstract}
Summary: This study compared the size, histology and morphology of coexisting neoplastic polyps found in colonoscopy, and evaluated the clustering of these polyps in patients with either colorectal carcinoma, cancerous polyps or benign adenomas. Patients were divided by their most malignant form of neoplasia: Group A included 58 patients with early invasive cancerous polyps, and Group B included 73 patients with in situ carcinoma within an adenoma. Group $\mathbf{C}$ consisted of 335 patients with benign adenomas, and Group D had 289 patients with colorectal carcinoma. There were no significant differences between the four groups regarding sex, age and ethnicity. The cancerous polyps were significantly larger than the benign polyps.

There were significantly $(P<0.01)$ more patients with multiple $(\geqslant 5)$ colonic lesions in Groups A or B than in Groups C or D. Coexisting polyps were much closer to the index growth, and demonstrated more severe dysplastic changes in the case of cancerous polyps than those associated with benign polyps or cancer.

Based on our data we speculate that cancerous polyps are not merely a middle link in adenoma-carcinoma sequence, but rather mark a subset of patients who are especially prone to develop neoplastic changes in their colonic mucosa. These patients should be included in a more strict colonoscopic surveillance programme.
\end{abstract}

\section{Introduction}

Carcinomatous elements may be found in $5 \%$ of colorectal adenomas, ${ }^{1,2}$ which are then termed cancerous or malignant polyps. They are divided into two subsets with different prognosis: (a) the carcinoma in situ, namely those confined to the mucosa, which practically do not have the ability to metastasize; (b) the invasive polyps, defined as adenomas, with a neoplastic process that extends beyond the muscularis mucosa or even beyond polyp boundaries. The question arises whether cancerous polyps represent an intermediate stage between benign adenomas and true cancers (benign $\rightarrow$ in situ $\rightarrow$ invasive $\rightarrow$ cancer), or constitute a special group of polyps with different characteristics. It may be assumed that if cancerous polyps were merely a link in the adenomacarcinoma sequence, then synchronous adenomas

Correspondence: A. Pines, M.D., Department of Medicine 'T', Ichilov Hospital, Tel-Aviv, Israel 64239 Accepted: 12 February 1991 which appear in patients with either benign adenomas or cancerous polyps, or with carcinomas, would have similar characteristics. In this study we examined the synchronous adenomas of patients with different types of colorectal neoplasia.

\section{Patients and methods}

This retrospective study included four groups of patients, all of which were diagnosed by total colonoscopy during 1978-1988. Group A consisted of 58 patients with early invasive cancerous polyps, namely, polyps containing a focus of carcinoma which extends into or beyond the muscularis mucosa, but the polypectomy resection margin free of tumoral infiltration. Group B included 73 patients with adenomas containing a focus of severe dysplasia (carcinoma in situ). While examining the polyps of Group A and Group B 
altogether, they were termed cancerous polyps. Polypoid carcinoma was not considered a cancerous polyp. Three hundred and fifty-three patients with benign adenomas (mild or moderate dysplasia) formed Group C, and 289 with colorectal carcinoma constituted Group D. Patients with several types of polyps were classified by their most malignant lesion (invasive $>$ in situ $>$ benign). Two independent pathologists examined the polyps, and used the WHO classification. ${ }^{3}$ In cases of disagreement, slides were shown to a third pathologist who made the final assessment. Demographic and clinical data were obtained from the outpatient clinic and hospital records. The following variables were compared: tumour size $(<0.9,1-1.9, \geqslant 2 \mathrm{~cm})$, morphology (sessile or pedunculated); histology (tubular, mixed tubovillous, villous); number of lesions (single, $2-4, \geqslant 5$ polyps); and the smallest distance in centimetres between an index lesion and other coexisting growth, measured by additional $5 \mathrm{~cm}$ marks on the colonoscope. Two-tailed Chi-Square and Fisher's Exact tests were used for the statistical analyses.

\section{Results (Table I)}

There were no differences between the four groups regarding age, sex and ethnicity of patients. Syn- chronous adenomas were found in 31 patients (54\%) of Group A (invasive polyps) having 68 coexisting lesions and in 42 patients $(58 \%)$ of Group B (carcinoma in situ) with 116 coexisting adenomas. The corresponding figures for Group C (benign adenomas) were 138 patients $(41 \%)$ with 419 adenomas, and for Group D (cancer) - 80 patients $(28 \%)$ with 158 adenomas. Among patients with cancerous polyps there were significantly fewer patients with a single colonic lesion than in Groups $\mathrm{C}$ or $\mathrm{D}\left(P<0.006\right.$ and $P<10^{-6}$, respectively). Furthermore, in Groups A or B there were significantly more persons with $\geqslant 5$ polyps than in Groups $\mathrm{C}$ or $\mathrm{D}(P<0.01$ for both). Coexisting polyps were much closer to the index growth in the case of cancerous polyps - if taken as a whole than those associated with benign adenomas or cancer $(P<0.02)$. However, a statistical significance was not reached in most distance analyses when Groups A or B were examined separately, probably because of the relatively small numbers of synchronous polyps included in these groups. Although cancerous polyps, the invasive ones in particular, were significantly larger than the adenomas of Group C $(P<0.001)$, there were no size differences among the coexisting adenomas of Groups A, B or D as compared to Group C. Cancer patients had more synchronous villous adenomas than patients of all other groups, but the distribu-

Table I Clinical parameters of synchronous adenomas in patients with cancerous polyps, benign adenomas ant cancer

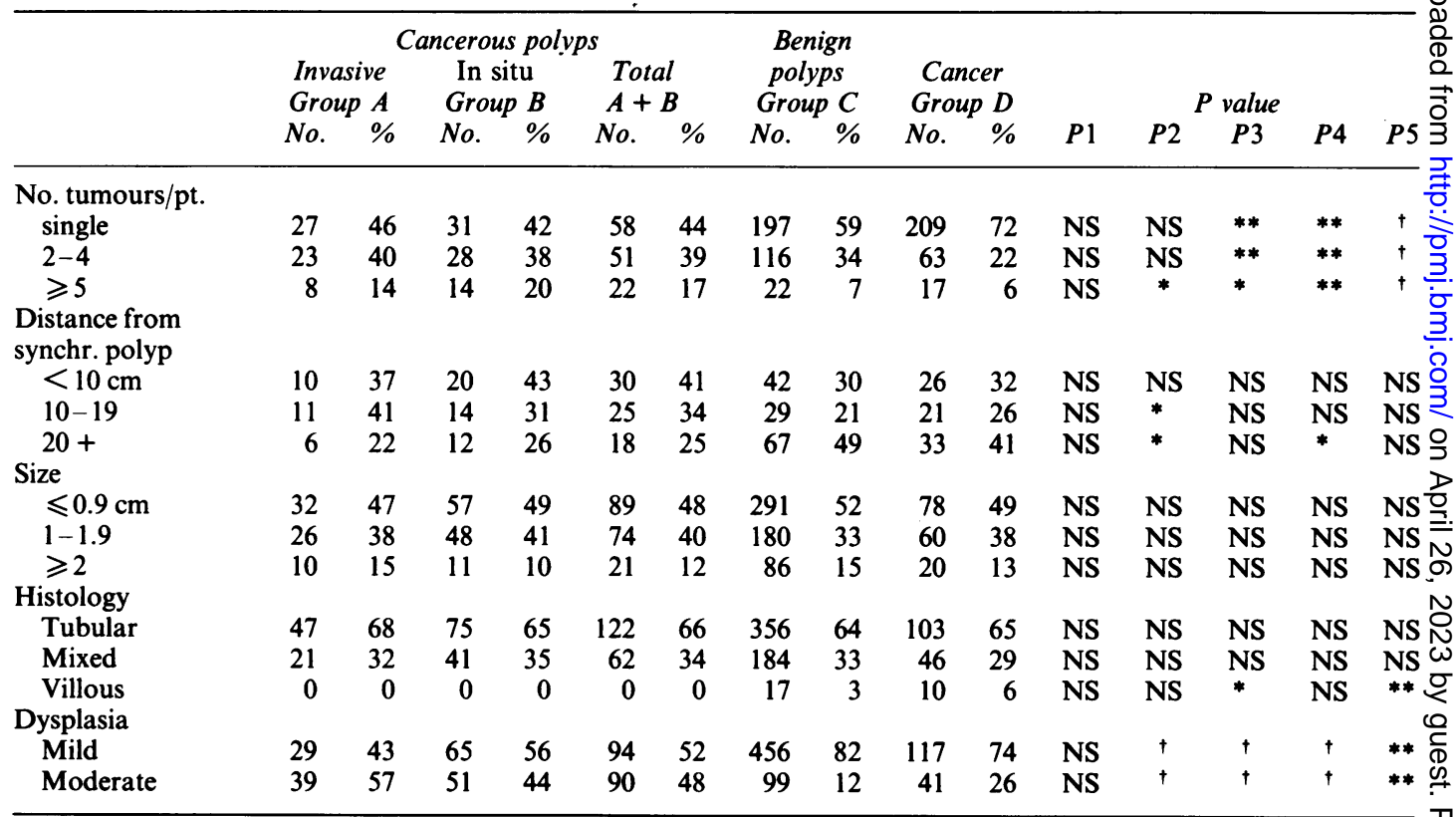

Totals may vary due to exclusion of persons with missing data. $P 1=$ comparison of Groups $A$ and $B ; P 2=$ comparison , Groups $A$ and $C ; P 3=$ comparison of Groups A and D; $P 4=$ comparison of Groups B and C; $P$ 5 = comparison 1 Groups B and D; NS = not significant; ${ }^{*}=P<0.05 ;{ }^{* *}=P<0.01 ;^{\dagger}=P<0.001$. 
tion of tubular or mixed adenomas was similar among all groups. The synchronous adenomas in Groups A and B demonstrated significantly more severe dysplastic changes than their counterparts in Group C or D. The level of dysplasia was not influenced by the distance from the cancerous polyps.

\section{Discussion}

Assuming that the appearance of a focus containing cancerous cells within an adenoma is just an intermediate phase in the malignant evolution of a certain benign polyp into cancer, this would indicate that the characteristics of other coexisting adenomas, if present, would resemble those in persons having benign adenomas only. If this is not found, one may suggest that cancerous polyps perhaps represent a separate subset of patients with colorectal neoplasia. Indeed, our results showed that patients with cancerous polyps had a significantly higher rate of synchronous adenomas when compared to patients with benign adenomas or carcinomas. These synchronous tumours tended to be situated close to the cancerous polyps, and demonstrated higher grade of dysplasia.

In the present series, over $50 \%$ of the patients with cancerous polyps had at least one additional lesion, and $18 \%$ had 5 or more colorectal neoplasms. The incidence of synchronous polyps in surgical material from patients with resected colon cancer ranged from 15 to $50 \%{ }^{4-8}$ Slater et al. ${ }^{4}$ who found associated polyps in $30 \%$ of 591 specimens, noted that the length of intestines resected and thus evaluated for polyps ranged between $20-34 \mathrm{~cm}$. Ekelund and Lindstrom ${ }^{8}$ described a series of 960 patients with colorectal carcinoma of whom $22 \%$ had concomitant polyps. The investigators divided the colon into 6 segments and found that the benign neoplasms tended to cluster in the vicinity of the carcinomas. The phenomenon of clustering of synchronous neoplasms raises the question whether there is an influence of one tumour on the location of another tumour. Our data indicate that over $40 \%$ of the synchronous tumours were found within the same segment of the cancerous polyp, namely in a distance of less than $10 \mathrm{~cm}$. Moreover, taking into consideration that the growth of a carcinoma might destroy adjacent polyps could mean that the above mentioned rate should be even higher. Recently, Cappell and Forde ${ }^{9}$ reported on 426 patients with multiple colon polyps. They found that both neoplastic and hyperplastic polyps tended to appear in clusters.

A major problem in the understanding of colorectal carcinogenesis is the fact that, in a colon which contains several adenomas, most remain small and benign while only one or two become highly dysplastic. There is still no clear explanation for this phenomenon, although luminal or humoral factors ${ }^{10}$ and chromosomal alterations ${ }^{11}$ have been proposed as pathogenetic mechanisms for tumour progression. We believe that cancerous polyps appear in patients whose colonic mucosa is more susceptible to neoplastic transformation compared to patients with benign polyps only or with carcinoma. The higher incidence of neoplasms around the cancerous polyps could mean either that these polyps induce local tumour promoting changes in their vicinity, or that cancerous polyps develop mainly in certain colonic areas characterized by mucosal predisposition for cancer development.

Hill et al. ${ }^{10}$ proposed a pathogenetic model based on three factors involved in carcinogenesis. Factor E1 produces small adenomas, factor E2 makes the small adenomas bigger and more dysplastic, and factor $\mathrm{C}$ induces carcinoma in a high proportion of adenomas. A change in the relative amounts of these factors may lead to different expressions concerning neoplastic alterations within the mucosa. One might speculate that patients with cancerous polyps are more likely to have increased activation of all three factors. In this situation we would expect multiple colon lesions, both benignand malignant, and more dysplastic changes including cancerous foci within adenomas. Our data seem to support this hypothesis. Furthermore, if this activity is more pronounced at certain locations, it could fit our observation that cancerous polyps were found to be close to other colon neoplasms. Another result which favours this hypothesis was the higher rate of severe dysplastic changes found in the coexisting benign polyps in Groups A and B. Similar findings were reported by Kalus $^{12}$ in cancer patients: $47 \%$ of synchronous polyps in the same segment showed severe dysplasia in contrast to an incidence of $6 \%$ for severe dysplastic changes found among patients with benign adenomas only.

\section{Conclusions}

In view of all these data we believe that cancerous polyps may not be just the middle link in the adenoma-carcinoma sequence, but rather constitute a marker for a special subset of patients who are more prone to the development of malignant transformation in their colonic mucosa. Hence, the finding of a cancerous polyp should allocate the patient into a higher risk group, and necessitate a more close and frequent colonoscopic surveillance. 


\section{References}

1. Morson, B.C. The Pathogenesis of Colorectal Cancer. W.B. Saunders, Philadelphia, 1978, pp. 1-164.

2. Shinya, H. \& Wolff, W.I. Morphology, anatomic distribution and cancer potential of colonic polyps. An analysis of 7000 polyps endoscopically removed. Ann Surg 1979, 190: $679-683$.

3. World Health Organization. Histological typing of intestinal tumours. In: Morson, B.C., Sobin, L.H. (eds) International Histological Classification of Tumours, Vol. 15. WHO, Geneva, 1976.

4. Slater, G., Fleshner, P. \& Aufses, A.H. Colorectal cancer location and synchronous adenomas. Am J Gastroenterol 1988, 83: 832-836.

5. Muto, T., Bussey, H.J.R. \& Morson, B.C. The evaluation of cancer of the colon and rectum. Cancer 1975, 36: 2251-2273.

6. Chu, D.Z.J., Giacco, G., Martin, R.G. et al. The significance of synchronous carcinomas and polyps in the colon and rectum. Cancer 1986, 57: 445-450.
7. Bat, L., Neumann, G. \& Shemesh, E. The association of synchronous neoplasms with occluding colorectal cancer. Dis Colon Rectum 1985, 28: 149-151.

8. Ekelund, G. \& Lindstrom, C. Histopathological analysis of benign polyps in patients with carcinoma of the colon and rectum. Gut 1974, 15: 654-663.

9. Cappell, M.S. \& Forde, K.A. Spatial clustering of multiple hyperplastic, adenomatous, and malignant polyps in individual patients. Dis Colon Rectum 1989, 32: 641-652.

10. Hill, M.J., Morson, B.C. \& Bussey, H.J.R. Aetiology of adenoma-carcinoma sequence in large bowel. Lancet 1978, i: 245-247.

11. Vogelstein, B., Fearon, E.C., Hamilton, S.R. et al. Genetic alterations during colorectal-tumor development. $N$ Engl $J$ Med 1988, 319: 525-532.

12. Kalus, M. Carcinoma and adenomatous polyps of the colon and rectum in biopsy and organ tissue culture. Cancer 1972, 30: $972-982$. 